\title{
ADAPTIVE CONTROL OF ISOTHERMAL REACTOR WITH COMPLEX REACTION
}

\author{
Jiri Vojtesek, Frantisek Gazdos and Petr Dostal \\ Faculty of Applied Informatics \\ Tomas Bata University in Zlin \\ Nad Stranemi 4511, 76005 Zlin, Czech Republic \\ E-mail: \{vojtesek,gazdos,dostalp\}@fai.utb.cz
}

\section{KEYWORDS}

CSTR, Adaptive control, Polynomial approach, Poleplacement method, Recursive identification

\begin{abstract}
The role and importance of the simulation rises with the increasing speed of computers and simulation tools provided nowadays. Safety and less money and time demands gives a computer simulation big advantage over the experiments on a real system or its model. The paper deals with simulation of an adaptive control on a nonlinear system represented by a Continuous Stirred Tank Reactor (CSTR). This system is mathematically described by a set of Ordinary Differential Equations (ODE) which are first solved numerically to obtain steady-state and dynamic behaviour of the system. The adaptive control is based on the recursive identification of an External Linear Model (ELM) as a representation of the originally nonlinear system. The polynomial approach together with the pole-placement method gives sufficient control results although the system has negative control properties.
\end{abstract}

\section{INTRODUCTION}

The Continuous Stirred Tank Reactors (CSTRs) are often used in chemical or biochemical industry for their good control properties. The flow of reactant is fluent and it can be in most cases easily controlled by the volumetric flow rate (Ingham et al. 2000).

This paper deals with adaptive control of the isothermal CSTR with complex reaction inside the tank (Russell and Denn 1972). This system is mathematically described by the set of five Ordinary Differential Equations (ODE) which are solved numerically via well-known Runge-Kutta's standard method (Johnston 1982). The steady-state and dynamic analyses presented in (Zelinka et al. 2006) results in optimal working point and the choice of the External Linear Model (ELM) which represents the originally nonlinear CSTR.

The polynomial approach together with a poleplacement method and spectral factorization are used for design of a controller. Parameters of the controller are periodically recomputed according to the parameters of the ELM which are estimated recursively during the control (Bobal et al. 2005). The ordinary recursive least squares method (Fikar and Mikleš 1999) was used for the parameter estimation.

All proposed control strategies were verified by and simulations in mathematical software MATLAB 6.5.

\section{MODEL OF THE PLANT}

The reactor under the consideration is an Isothermal reactor with complex reaction (Ingham et al. 2000). Reactions inside the reactor can be described by following relations:

$$
\begin{aligned}
& \mathrm{A}+\mathrm{B} \stackrel{\mathrm{k}_{1}}{\longrightarrow} \mathrm{X} \\
& \mathrm{B}+\mathrm{X} \stackrel{\mathrm{k}_{2}}{\longrightarrow} \mathrm{Y} \\
& \mathrm{B}+\mathrm{Y} \stackrel{\mathrm{k}_{3}}{\longrightarrow} \mathrm{Z}
\end{aligned}
$$

These reactions have sequential $(\mathrm{A} \rightarrow \mathrm{X} \rightarrow \mathrm{Y} \rightarrow \mathrm{Z})$ as well as parallel characteristics $(\mathrm{B} \rightarrow \mathrm{X}, \mathrm{B} \rightarrow \mathrm{Y}, \mathrm{B} \rightarrow$ $\mathrm{Z}$ ). Some simplifications must be introduced because of complexity of this system. We assume that the reactant inside the tank is perfectly mixed and volume of the reactant is constant during the reaction. The mathematical model of the system is then derived from the material balances inside the reactor:

$$
\begin{gathered}
\frac{d c_{A}}{d t}=\frac{q}{V}\left(c_{A 0}-c_{A}\right)-k_{1} \cdot c_{A} \cdot c_{B} \\
\frac{d c_{B}}{d t}=\frac{q}{V}\left(c_{B 0}-c_{B}\right)-k_{1} \cdot c_{A} \cdot c_{B}- \\
-k_{2} \cdot c_{B} \cdot c_{X}-k_{3} \cdot c_{B} \cdot c_{Y} \\
\frac{d c_{X}}{d t}=\frac{q}{V}\left(c_{X 0}-c_{X}\right)+k_{1} \cdot c_{A} \cdot c_{B}-k_{2} \cdot c_{B} \cdot c_{X} \\
\frac{d c_{Y}}{d t}=\frac{q}{V}\left(c_{Y 0}-c_{Y}\right)+k_{2} \cdot c_{B} \cdot c_{X}-k_{3} \cdot c_{B} \cdot c_{Y} \\
\frac{d c_{Z}}{d t}=\frac{q}{V}\left(c_{Z 0}-c_{Z}\right)+k_{3} \cdot c_{B} \cdot c_{Y}
\end{gathered}
$$

This set of nonlinear ODE describes behaviour of the state variables which are in this case concentrations of components $A, B, X, Y$ and $Z-c_{A}, c_{B}, c_{X}, c_{Y}$ and $c_{Z}$ in time $t$. Under the simplifications introduced above, this model belongs to the class of nonlinear lumpedparameters systems.

In the set (2) - (6) $q$ denotes volumetric flow rate, $V$ is volume of the tank, $k$ describes rate constants and $c$ are concentrations. Numerical subscripts 1,2 and 3 represent reaction steps. 
Table 1: Parameters of the reactor

\begin{tabular}{|ll|}
$k_{1}=5 \times 10^{-4} \mathrm{~m}^{3} \cdot \mathrm{kmol}^{-1} \cdot \mathrm{s}^{-1}$ & $k_{2}=5 \times 10^{-2} \mathrm{~m}^{3} \cdot \mathrm{kmol}^{-1} \cdot \mathrm{s}^{-1}$ \\
$k_{3}=2 \times 10^{-2} \mathrm{~m}^{3} \cdot \mathrm{kmol}^{-1} . \mathrm{s}^{-1}$ & $V=1 \mathrm{~m}^{3}$ \\
$c_{A 0}=0.4 \mathrm{kmol} . \mathrm{m}^{-3}$ & $c_{B 0}=0.6 \mathrm{kmol} . \mathrm{m}^{-3}$ \\
$c_{X 0}=0 \mathrm{kmol} . \mathrm{m}^{-3}$ & $c_{Y 0}=0 \mathrm{kmol} \cdot \mathrm{m}^{-3}$ \\
$c_{Z 0}=0 \mathrm{kmol} . \mathrm{m}^{-3}$ & \\
\hline
\end{tabular}

Schematic representation of the reactor can be seen in Figure 1 and technological parameters and constans are shown in Table 1.

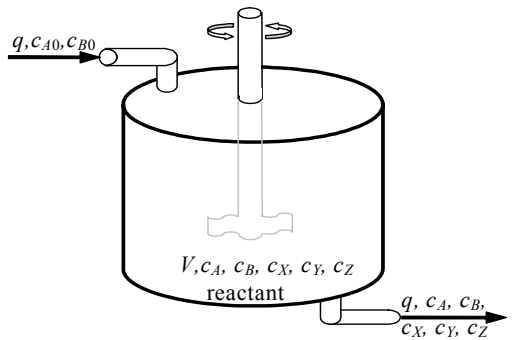

Figure 1: Continuous Stirred Tank Reactor

\section{STEADY-STATE AND DYNAMIC ANALYSES}

\section{Steady-state Analysis}

The steady-state analysis is the first step in the preparation of the optimal controller. It shows behaviour of the system in the steady-state, mathematically for $t \rightarrow \infty$ and results in optimal working point in the sense of maximal effectiveness and concentration yield.

The static characteristic for different input volumetric flow rate $q$ presented in (Zelinka et al. 2006) shows that this system has nonlinear behaviour. The volumetric flow rate of the reactant $q^{s}=0.0001 \mathrm{~m}^{3} \cdot \mathrm{s}^{-1}$ was chosen as a working point for the dynamic analysis and control. Steady-state values of the state variables in this working point are:

$$
\begin{gathered}
c_{A}^{s}=0.2407 \text { kmol.m } \mathrm{m}^{-3} \quad c_{B}^{s}=0.1324 \mathrm{kmol} . \mathrm{m}^{-3} \\
c_{X}^{s}=0.0024 \mathrm{kmol} . \mathrm{m}^{-3} \quad c_{Y}^{s}=0.0057 \mathrm{kmol} . \mathrm{m}^{-3} \\
c_{Z}^{s}=0.1513 \mathrm{kmol} . \mathrm{m}^{-3}
\end{gathered}
$$

\section{Dynamic Analysis}

Dynamic behaviour of the system is obtained after a change of the input volumetric flow rate $q$. Figure 2 displays output responses of concentration $c_{z}$ to four step changes of the input variable $q(\Delta q= \pm 100 \%$, $\pm 50 \%$ of $q$ ) related to its steady-state value.

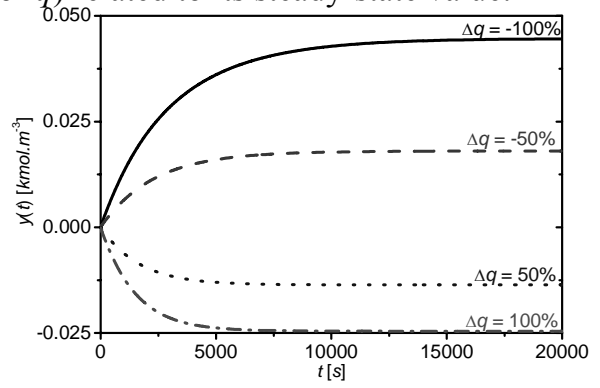

Figure 2: Time response of the output $y$ for various step changes of the input volumetric flow rate $q$
This recomputation is because of better display of the output response - steady-state values are initial conditions for numerical solving of the set of ODE (2)(6) and consequently the graph starts in zero, i.e.

$$
y(t)=c_{Z}(t)-c_{Z}^{s}
$$

Output responses shown in Figure 2 shows that this output can be mathematically represented by the second order transfer function with relative order one:

$$
G(s)=\frac{b(s)}{a(s)}=\frac{b_{1} s+b_{0}}{a_{2} s^{2}+a_{1} s+a_{0}}
$$

where $s$ is a complex variable, $a_{0,1,2}$ and $b_{1,0}$ are coefficients and $b_{0}, b_{1}$ differs in sign for non-minimum phase systems. The transfer function with the first order or the second order with relative order two, i.e.

$$
G(s)=\frac{b_{0}}{a_{1} s+a_{0}}, G(s)=\frac{b_{0}}{a_{2} s^{2}+a_{1} s+a_{0}}
$$

could be used too, but simulation experiments have shown that transfer function (9) is more suitable because it can include non-minimum behaviour which cannot be seen from the dynamic behaviour but it can occur in some working points during the control. For further computation we expect $a_{2}=1$.

\section{ADAPTIVE CONTROL}

Adaptive control is one of the dynamically expanding modern control approaches which can be used for systems with time delays, nonlinear systems, nonminimum phase systems etc. These systems have negative properties from the control point view and control responses with classical P, PI, PID etc. controllers can result in unstable or non-optimal responses.

The adaptive approach used in this work is based on a choice of the External Linear Model (ELM) of the nonlinear process, parameters of which are estimated recursively and the controller parameters are then recomputed in every step according to the estimated parameters of the ELM (Bobal et al. 2005).

The resultant controller works in continuous-time and in our case its structure corresponds to the structure of the real PID controller but its parameters vary according to the actual working point.

\section{External Linear Model(ELM)}

As it is written above, the originally nonlinear system is replaced by the External Linear Model (ELM). We have chosen the transfer function described by the equation (9) from the previous chapter, i.e.

$$
G(s)=\frac{Y(s)}{U(s)}=\frac{b(s)}{a(s)}=\frac{b_{1} s+b_{0}}{a_{2} s^{2}+a_{1} s+a_{0}}
$$

This transfer function fulfils a condition of properness $\operatorname{deg} b \leq \operatorname{deg} a$.

The ELM can be in continuous time or discrete time form. In this work, $\delta$-model was used as an ELM. This 
model belongs to the class of discrete models but its properties are different from the classical discrete model in the Z-plain. If we want to convert $Z$-model to $\delta$-model, we must introduce a new complex variable $\gamma$ computed as (Mukhopadhyay et al. 1992)

$$
\gamma=\frac{z-1}{\alpha \cdot T_{v} \cdot z+(1-\alpha) \cdot T_{v}}
$$

We can obtain infinitely many models for optional parameter $\alpha$ from the interval $0 \leq \alpha \leq 1$ and a sampling period $T_{v}$, however a forward $\delta$-model was used in this work which has $\gamma$ operator computed via

$$
\alpha=0 \Rightarrow \gamma=\frac{z-1}{T_{v}}
$$

The ELM (9) is then rewritten to the general differential equation

$$
a^{\prime}(\delta) y\left(t^{\prime}\right)=b^{\prime}(\delta) u\left(t^{\prime}\right)
$$

where $t^{\prime}$ denotes discrete time and $\delta$ is the operator defined according to (12). With decreasing value of the sampling period $T_{v}$ parameters of polynomials $a^{\prime}(\delta)$ and $b^{\prime}(\delta)$ approach the parameters of the continuous-time model (Stericker and Sinha 1993).

The relation for the actual output is derived from the (13) as

$$
\begin{aligned}
y_{\delta}(k)= & -a_{1} y_{\delta}(k-1)-a_{0} y_{\delta}(k-2)+ \\
& +b_{1} u_{\delta}(k-1)+b_{0} u_{\delta}(k-2)
\end{aligned}
$$

where $y_{\delta}$ is the recomputed output to the $\delta$-model:

$$
\begin{aligned}
& y_{\delta}(k)=\frac{y(k)-2 y(k-1)+y(k-2)}{T_{v}^{2}} \\
& y_{\delta}(k-1)=\frac{y(k-1)-y(k-2)}{T_{v}} \\
& y_{\delta}(k-2)=y(k-2) \\
& u_{\delta}(k-1)=\frac{u(k-1)-u(k-2)}{T_{v}} \\
& u_{\delta}(k-2)=u(k-2)
\end{aligned}
$$

where $T_{v}$ is a sampling period, the data vector is then

$$
\begin{array}{r}
\phi^{T}(k-1)=\left[-y_{\delta}(k-1),-y_{\delta}(k-2), \ldots\right. \\
\left.\ldots, u_{\delta}(k-1), u_{\delta}(k-2)\right]
\end{array}
$$

and the vector of estimated parameters

$$
\hat{\boldsymbol{\theta}}^{T}(k)=\left[\hat{a}_{1}, \hat{a}_{0}, \hat{b}_{1}, \hat{b}_{0}\right]
$$

can be computed from the ARX (Auto-Regressive eXtrogenous) model

$$
y_{\delta}(k)=\theta_{\delta}^{T}(k) \cdot \varphi_{\delta}(k-1)
$$

by some of the recursive least squares methods.

Simulation experiments have shown that there is no need to use forgetting factors because the resulted output response is the same for Ordinary Recursive Least Squares (ORLS) method and its modifications. The ORLS method is one of the basic identification methods and it can be formally described by the set of equations (Fikar and Mikles 1999):

$$
\begin{gathered}
\varepsilon(k)=y(k)-\boldsymbol{\varphi}^{T}(k) \cdot \hat{\boldsymbol{\theta}}(k-1) \\
\gamma(k)=\left[1+\boldsymbol{\varphi}^{T}(k) \cdot \mathbf{P}(k-1) \cdot \boldsymbol{\varphi}(k)\right]^{-1} \\
\boldsymbol{L}(k)=\gamma(k) \cdot \mathbf{P}(k-1) \cdot \boldsymbol{\varphi}(k) \\
\mathbf{P}(k)=\mathbf{P}(k-1)-\gamma(k) \cdot \mathbf{P}(k-1) \cdot \boldsymbol{\varphi}(k) . \\
\cdot \boldsymbol{\varphi}^{T}(k) \cdot \mathbf{P}(k-1) \\
\hat{\boldsymbol{\theta}}(k)=\hat{\boldsymbol{\theta}}(k-1)+\boldsymbol{L}(k) \varepsilon(k)
\end{gathered}
$$

Where $\varepsilon$ denotes a prediction error and $\mathbf{P}$ is a covariance matrix.

\section{Configuration of the Controller}

The configuration with one degree-of-freedom (1DOF) was used for the control system set-up. This form has a controller in the feedback part - see Figure 3.

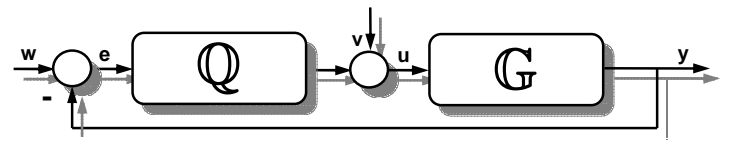

Figure 3: 1DOF control configuration

The block $G$ in the Figure 3 represents the transfer function of the plant (9), $w$ is the wanted value (reference signal), $e$ stands for the control error $(e=w-$ $y), v$ is a disturbance, $u$ is used for the control variable and $y$ denotes the controlled output. Block $Q$ is a transfer function of the controller which ensures stability, asymptotic tracking of the reference signal and load disturbance attenuation and it can be described by the polynomials in $s$-plain as

$$
Q(s)=\frac{q(s)}{s \cdot \tilde{p}(s)}
$$

where degrees of the polynomials are computed from

$$
\operatorname{deg} q(s)=\operatorname{deg} a(s), \operatorname{deg} \tilde{p}(s) \geq \operatorname{deg} a(s)-1
$$

and parameters of the polynomials $\tilde{p}(s)$ and $q(s)$ are computed from a Diophantine equation (Kucera 1993):

$$
a(s) \cdot s \cdot \tilde{p}(s)+b(s) \cdot q(s)=d(s)
$$

Polynomials $a(s)$ and $b(s)$ are known from the recursive identification and the polynomial $d(s)$ on the right side of (22) is an optional stable polynomial. Roots of this polynomial are called poles of the closed-loop and their position affects quality of the control. The degree of the polynomial $d(s)$ is in this case

$$
\operatorname{deg} d(s)=\operatorname{deg} a(s)+\operatorname{deg} \tilde{p}(s)+1
$$

A choice of the roots needs some a priory information about the system's behaviour. It is good to connect poles with the parameters of the system via spectral factorization.

The polynomial $d(s)$ is then of the fourth degree and it can be rewritten for aperiodical processes to the form

$$
d(s)=n(s) \cdot(s+\alpha)^{\operatorname{deg} d-\operatorname{deg} n}
$$


where $\alpha>0$ is an optional coefficient reflecting closedloop poles and stable polynomial $n(s)$ is obtained from the spectral factorization of the polynomial $a(s)$

$$
n^{*}(s) \cdot n(s)=a^{*}(s) \cdot a(s)
$$

As written above, the second order transfer function with the relative order one was chosen as an ELM of the system. As a result, degrees of the polynomials are computed via (21): $\operatorname{deg} q(s)=2$ and $\operatorname{deg} \tilde{p}(s)=1$ which means, that the transfer function (20) is

$$
\tilde{Q}(s)=\frac{q_{2} s^{2}+q_{1} s+q_{0}}{s \cdot\left(p_{1} s+p_{0}\right)}
$$

and the polynomial $d(s)$ is from (24) of the fourth degree and it could be chosen as

$$
d(s)=n(s) \cdot(s+\alpha)^{2}
$$

where $\alpha>0$ is an optional parameter which reflects some of the closed-loop roots. Parameters of the polynomial $n(s)$ which are computed from the spectral factorization are defined as:

$$
n_{0}=\sqrt{a_{0}^{2}}, n_{1}=\sqrt{a_{1}^{2}+2 n_{0}-2 a_{0}}
$$

\section{Simulation results}

Simulation experiments of the adaptive control theoretically described in previous sections were done in the mathematical software Matlab, version 6.5.1.

As it is written in the dynamic analysis part, the concentration of the output $Z, c_{Z}$, subtracted from its steady-state value $c_{Z}^{s}$ was chosen as the output variable $y$ and the change of the input volumetric flow rate, $\Delta q$ in $\%$, was set as the control input variable $u$, i.e.

$$
\begin{aligned}
& y(t)=c_{Z}(t)-c_{Z}^{s}\left[\text { kmol.m }{ }^{-3}\right] \\
& u(t)=\frac{q(t)-q^{s}}{q^{s}} \cdot 100[\%]
\end{aligned}
$$

The control study was done for time $10000 s$ with the sampling period $T_{v}=2 \mathrm{~s}$. The input variable $u(t)$ was limited due to the physical realization to the bounds $u(t)=<-100 \% ;+100 \%>$. The initial parameters for identification were $-\hat{\boldsymbol{\theta}}^{T}=[0.1,0.1,0.1,0.1]$ and $\boldsymbol{P}_{i i}=$ $1 \cdot 10^{7}$ for $i=1, . ., 4$. The simulation study was peformed for a different position of the parameter $\alpha=0.005 ; 0.01$ and 0.05 and for five step changes of the reference signal $w(t)$ every $2000 \mathrm{~s}$. Results are shown in Figure 4 and Figure 5.

The results show that the control response is quicker with the increasing value of $\alpha$. On the other hand, the course of the input variable $u(t)$ is smoother and overshoots of the output variable are lower for lower values of $\alpha$. The overshoots of the output variable are a bit higher and changes of the input variable are big after the step change of the reference signal at the very beginning of the control. This is caused by the recursive identification which has small problems because of less amount of information at the beginning.

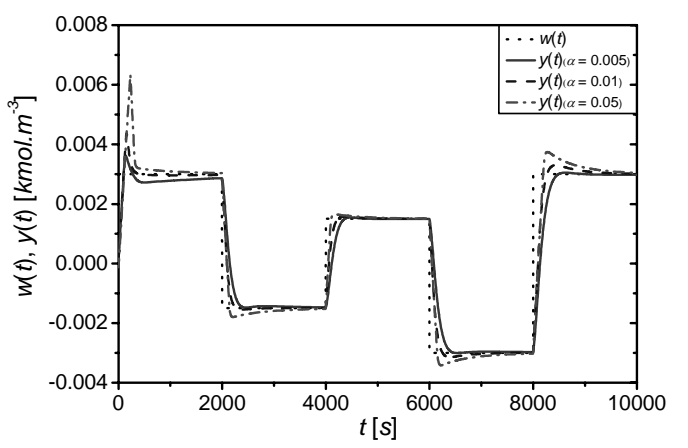

Figure 4: Output variable $y(t)$ for various values of

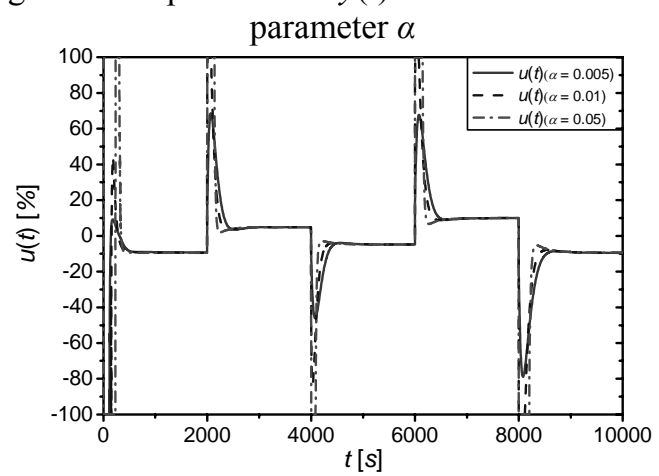

Figure 5: Input variable $u(t)$ for various values of parameter $\alpha$

The quality of the control was evaluated by the quality criteria $S_{u}$ and $S_{y}$ computed for a time interval as

$$
\begin{aligned}
& S_{u}=\sum_{i=2}^{N}(u(i)-u(i-1))^{2} \\
& S_{y}=\sum_{i=1}^{N}(w(i)-y(i))^{2}, \text { for } N=\frac{T_{f}}{T_{v}}
\end{aligned}
$$

where $T_{f}$ is the final time - in this case $T_{f}=10000 \mathrm{~s}$.

Table 2: Criteria of control quality

\begin{tabular}{|c|r|l|}
\hline & \multicolumn{1}{|c|}{$S_{u}$} & $S_{y}$ \\
\hline$\alpha=0.005$ & 5812.4614 & 0.0040 \\
$\alpha=0.01$ & 49566.6667 & 0.0026 \\
$\alpha=0.05$ & 116646.6775 & 0.0031 \\
\hline
\end{tabular}

Results for all three simulation studies are shown in Table 2. As you can see, increasing value of the parameter $\alpha$ affects mainly changes of the action value $u(t)$ which is illustrated by the criterion $S_{u}$. On the other hand, value of the criterion $S_{y}$, which is related to the control error, is the best for $\alpha=0.01$ and nearly similar to the other values.

The Figure 6 and Figure 7 display course of the identified parameters during control. Lines clearly show that the chosen ordinary recursive least-squares method has no problem with the identification of the ELM except for the starting time - that is why the data in both figures are cut from time $200 s$ when we have enough information about the system. The Figure 7 shows that $b^{\prime}{ }_{0}$ has very small value and does not change significantly during the control which means that we could neglect it. 


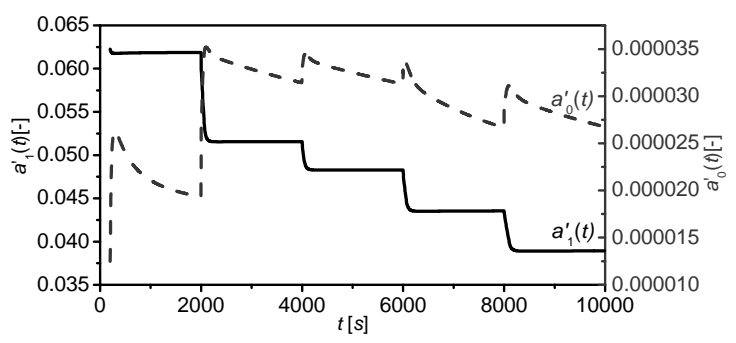

Figure 6: The course of the identified parameters $a^{\prime}{ }_{1}$ and $a_{0}{ }_{0}$ during the control

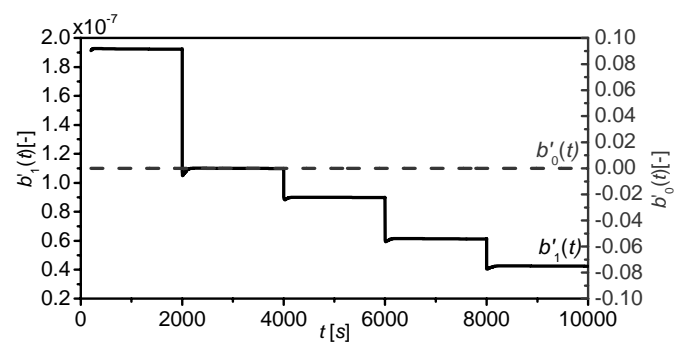

Figure 7: The course of the identified parameters $b_{1}$ and $b^{\prime}{ }_{0}$ during the control

\section{CONCLUSION}

The paper presents simulation results of the control of the nonlinear process represented by the isothermal CSTR with a complex reaction. The used adaptive control is based on the recursive identification of the external linear model of the nonlinear plant where estimated parameters of the system are used for computation of the controller's parameters via the polynomial approach and pole-placement method. Control results show that the proposed controller has good control responses although the system has nonlinear behaviour. Moreover, the output can be tuned by the choice of a different position of the parameter $\alpha$. Increasing value of the parameter results in higher speed of the control. However smaller values of this parameter produce lower overshoots and smoother course of the control action. The simulation studies show that there is no need to use forgetting factors in the recursive identification because the used ordinary recursive leastsquares method has no problem with the estimation.

\section{REFERENCES}

Bobal, V., Böhm, J., Fessl, J. Machacek, J 2005. Digital Selftuning Controllers: Algorithms, Implementation and Applications. Springer-Verlag London Limited

Fikar, M., Mikles J. 1999. System Identification. STU Bratislava

Ingham, J., Dunn, I. J., Heinzle, E., Přenosil, J. E. 2000. Chemical Engineering Dynamics. An Introduction to
Modeling and Computer Simulation. Second, Completely Revised Edition, VCH Verlagsgesellshaft, Weinheim.

Johnston, R. L. 1982. Numerical Methods. John Wiley \& Sons, New York

Kucera, V. 1993. Diophantine equations in control - A survey. Automatica, 29, 1361-1375

Mukhopadhyay, S., Patra, A.G., Rao, G.P. 1992. New class of discrete-time models for continuous-time systems. International Journal of Control, vol.55, 1161-1187

Russell, T. W. F. and Denn, M. M. 1972. Introduction to chemical engineering analysis. Wiley, McGraw-Hill

Stericker, D.L., Sinha, N.K. 1993. Identification of continuous-time systems from samples of input-output data using the $\delta$-operator. Control-Theory and Advanced Technology, vol. 9, 113-125

Zelinka, I., Vojtesek, J. and Oplatkova Z. 2006. Simulation Study of the CSTR Reactor for Control Purposes. In: Proc. of 20th European Conference on Modelling and Simulation ESCM 2006. Bonn, Germany, p. 479-482

\section{AUTHOR BIOGRAPHIES}

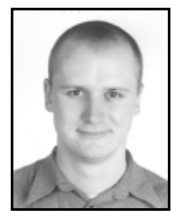

JIRI VOJTESEK was born in Zlin, Czech Republic and studied at the Tomas Bata University in Zlin, where he got his master degree in chemical and process engineering in 2002. Now he is about to finish his $\mathrm{Ph} . \mathrm{D}$. which is focused on Modern control methods for chemical reactors. You can conctact him on email address vojtesek@fai.utb.cz.

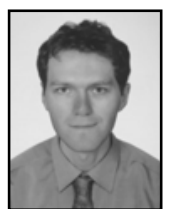

FRANTISEK GAZDOS was born in Zlin, Czech Republic, and graduated from the Technical University of Brno in 1999 with MSc. degree in Automation. He then followed studies of Technical cybernetics at the Tomas Bata University in Zlin, obtaining Ph.D. degree in 2004. Now he works as a senior lecturer (technological process control, state-space and algebraic approach to control system design) in the Department of Process Control, Faculty of Applied Informatics of the same University. E-mail: gazdos@fai.utb.cz.

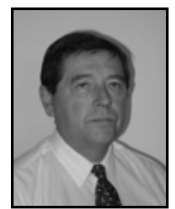

PETR DOSTAL studied at the Technical University of Pardubice, where he obtained his master degree in 1968 and $\mathrm{PhD}$. degree in Technical Cybernetics in 1979. In the year 2000 he became professor in Process Control. He is now head of the Department of Process Control. His research interest are modeling and simulation of continuous-time chemical processes, polynomial methods, optimal, adaptive and robust control. You can contact him on email address dostalp@fai.utb.cz. 